\title{
Hydrological model coupling with ANNs
}

\author{
R. G. Kamp ${ }^{1,2}$ and H. H. G. Savenije ${ }^{1}$ \\ ${ }^{1}$ Section of Water Resources, Delft University of Technology, Delft, The Netherlands \\ ${ }^{2}$ MX. Systems B.V., Rijswijk, The Netherlands
}

Received: 28 November 2006 - Published in Hydrol. Earth Syst. Sci. Discuss.: 6 December 2006

Revised: 31 August 2007 - Accepted: 21 November 2007 - Published: 4 December 2007

\begin{abstract}
There is an increasing need for model coupling. However, model coupling is complicated. Scientists develop and improve models to represent physical processes occurring in nature. These models are built in different software programs required to run the model. A software program or application represents part of the system knowledge. This knowledge is however encapsulated in the program and often difficult to access.

In integrated water resources management it is often necessary to connect hydrological, hydraulic or ecological models. Model coupling can in practice be difficult for many reasons related to data formats, compatibility of scales, ability to modify source codes, etc. Hence, there is a need for an efficient and cost effective approach to model-coupling. Artificial neural networks (ANNs) can be used as an alternative to replace a model and simulate the model's output and connect it to other models.
\end{abstract}

In this paper, we investigate an alternative to traditional model coupling techniques. ANNs are four different models: a rainfall runoff model, a river channel routing model, an estuarine salt intrusion model, and an ecological model. The output results of each model is simulated by a neural network that is trained on corresponding input and output data sets. The models are connected in cascade and their input and output variables are connected.

To test the results of the coupled neural network also a coupled system of four sub-system models has been set-up. These results have been compared to the results of the coupled neural networks. The results show that it is possible to train neural networks and connect these models. The results of the salt intrusion model was however not very accurate. It was difficult for the neural network to represent both short term (tidal) and long term (hydrological) processes.

Correspondence to: R. G. Kamp

(robert.kamp@mx-groep.nl)

\section{Introduction}

Water management influences many aspects of our modern life and has many inter-disciplinary fields. Water management deals not only with traditional tasks like safety and drainage, but also with our living standards, health and environment. This results in the demand for an integrated approach, inter-disciplinarity and coupled models for different sub-system elements (Koudstaal et al., 1992). Examples of hydrological models are rainfall-runoff models, free surface flow models and groundwater models. There already exist many coupled simulation programs in hydrology, for example the coupling of groundwater and surface flow models and the coupling of water quality and water quantity models. Another example is integration of water quality in urban waters and waste water treatments. Other fields related to water management are biological and ecological models. Model coupling of sub-system elements is necessary to answer complex questions.

The coupling of different models is intensive in time and costs. Segmented software development is most successful. Conquer and divide is a common way to solve complex problems. The negative side is that a large amount of energy is necessary to integrate two different types of computer models. From a software point of view software interfaces have difficulties with import/export tools, time steps, data formats and software versions. A real online time connection tends toward hybrid systems. To build on-line, tight couplings, fast connections or hybrid systems are necessary. It is only cost effective if it is used intensively. Another possibility is making components that can be plugged into one central framework. Many initiatives have been launched. One of the problems is that all stakeholders and future users must adopt and consequently implement one standard. This for example requires exact definition of all interfaces and results in less flexibility. Furthermore there are commercial and practical problems like product support, source update and legal issues.

Published by Copernicus Publications on behalf of the European Geosciences Union. 
On the one hand water management requires answers from different disciplines and on the other hand it is difficult to connect software programs. This research investigates the ability of neural networks to build hydrological models and to connect them. The models are designed in different software or software components as separate units. The focus is on model simulation and the coupling of model results. Many modeling experiments have shown it is possible to use neural networks to simulate hydrological or hydraulic models. In this paper a next step is made by using the ability of neural networks to build neural networks and to connect them. If it is possible to use neural networks to replace subsystem models trained on simulation results, this could be a valuable alternative in modeling practice. If a modeler needs to couple two different models he can build neural networks. The modeler can connect the models independent of the current application and it is not necessary to change software code or to connect the application to a computer framework. Normally those methods consume lots of time and resources.

The ANN is used as a simulator that operates as an interface between different computer models. The goal is to provide an alternative to traditional model coupling techniques. The assumption is that it is relatively easy to train a neural network on the outcome of a sub-system model. Neural network simulations are relatively fast compared to traditional simulations and coupling. However neural networks need additional training. A neural network simulates the output of sub-system models based on the input and output time series of these models. The output time series is the response of the system and reflects system processes. In so doing, the neural network learn the system processes. It is very difficult to program a system process or a conceptual model from physical phenomena directly in a neural network. It is not a common practice to translate mathematical relations one by one into a structure of hidden layers and transfer functions of a neuron. One example is that you cannot implement the unit-hydrograph directly into the elements of an ANN. Instead, the neural network is used to learn the relations between input and output data. Another restriction is that the neural network should be retrained after each adjustment to the hydrological model. If for example the area of a rainfall runoff model changes, the relation between a rainfall event and the river discharges changes as well. This results in a different output of the model and effects system response.

In this research, the focus is on four hydrological subsystem models in decoupled and coupled mode. The models cover different parts of a river system in a catchment area and are selected on a few criteria. They must be of equal complexity and they must have clear connection points to make it possible to verify the results. Another criterion is that it is possible to run the models in a coupled scenario to compare the neural network results. The models are described in Sect. 3.

\section{Methodology}

The goal of this paper is to show it is possible to couple four neural networks, representing hydrological models. An artificial neural network (ANN) is an interconnected group of artificial neurons that uses a mathematical model for information processing. In most cases a neural network is an adaptive system that changes its structure based on external information that flows through the network. In more practical terms neural networks are non-linear statistical data modeling tools. They can be used to model complex relationships between inputs and outputs (Haykin, 1999). The internal structure of a neural network consists of layers and neurons and weight values that control the transfer of internal signals. Neural network parameters, the weight values, have to be adjusted in an iterative training procedure. In this process, called training, the neural networks needs input that consists of corresponding model input and output. After training, the neural network can simulate or mimic the model.

The conventional way to couple simulations models is to connect software applications or to attach models to a global framework. The term model includes both the actual model, as one representation of a real natural system, as well as the model concept. This is the mathematical formulation of processes. Hydrological models are defined as models that simulate the water cycle. Finally the term model coupling itself is defined as coupling of distinct existing models that were developed to simulate processes in one system. Coupling in the present context mainly means coupling via exchange variables rather than directly coupling process equations and code. In this methodology the focus is on loose coupling. Tight and loose coupling can be defined by a communication/computation ratio. For a programmer loose coupling means a choice for modular design. The advantage is the ability to reuse a model and to build a complex system easily. However, modularity often comes at the price of reduced performance. With this methodology we try to use neural networks to connect the models without using the software application but by using the input and output data of the models. This is a loose connection.

A simulation model is a set of algorithms and mathematical rules that represent physical processes. A model is used to design or predict part of reality. To build a model it is necessary to describe a specific part of reality by a system. For example a modeler describes a river by several river sections with a certain profile. It is also important to define several model parameters. This might for example be the friction coefficient or infiltration capacity. After calibration of a model the user can calculate for example a river discharge or a water level. After calibration and validation the system design is finished. This is a situation in which a certain input data set results in a fixed output data. In other words, if the system stays the same, the relation between input and output is also the same. It can become a complex relation, but the system response is fixed. 

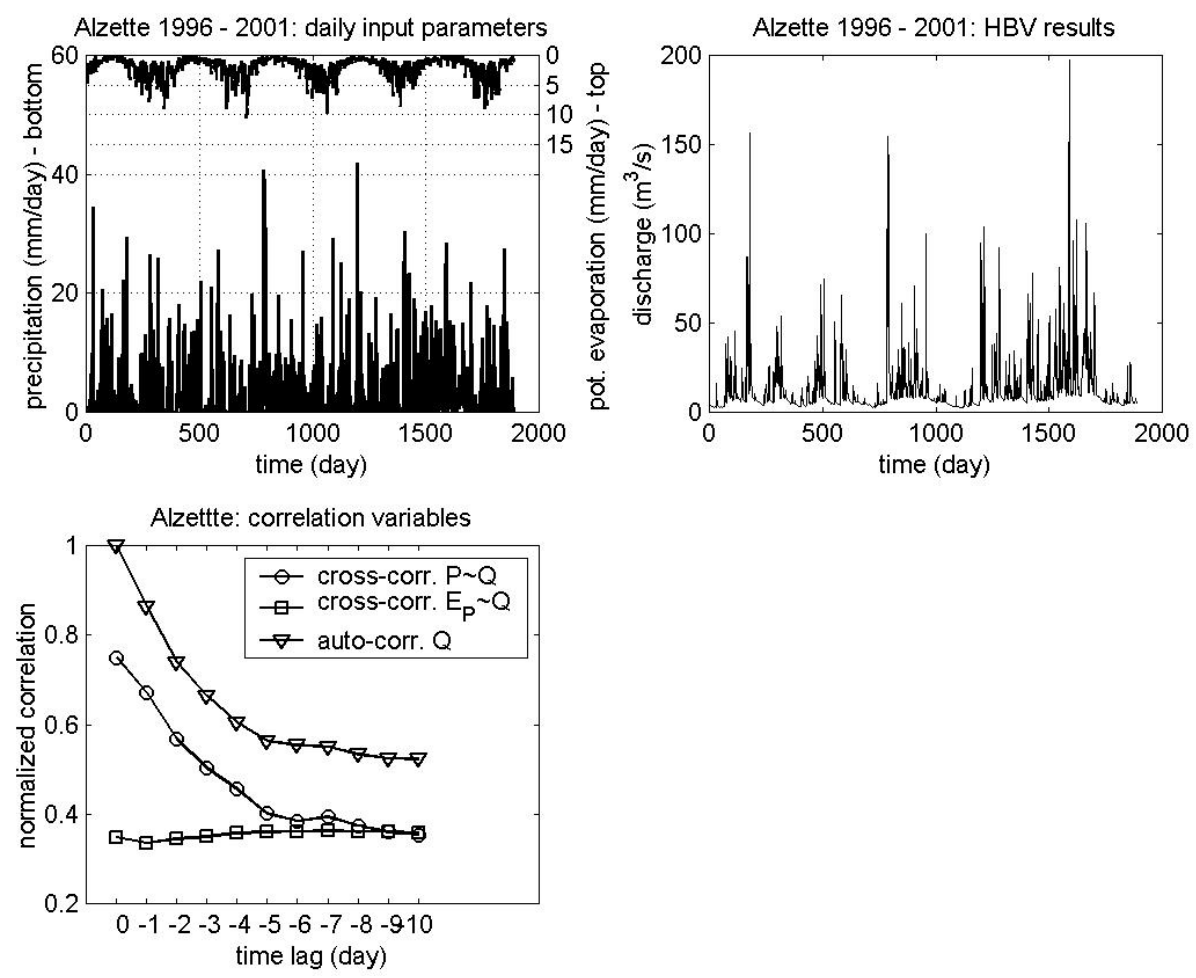

Fig. 1. Alzette basin RR-model, input data (upper left), neural network prediction (right), correlation input variables (down left).

This is also a characteristic of a neural network. Neural network are able to simulate complex input and output relations. The structure of a neural network can be complex. A neural network can function as a good universal function approximation. In this method the neural network will simulate the output of a single computer model based on the computer model's input parameters. The idea behind this is that all the system knowledge and physical processes are reflected in the system response.

The approach in this paper is to simulate four separate hydrological and hydraulic models in a decoupled and a coupled scenario. The decoupled scenario concerns the performance in emulating each of the four models and are useful to test the trained ANNs. The coupled scenario concerns the performance of the coupled situation, which is the goal of this paper. The models are coupled in a cascading order that globally follows the water stream in a river catchment. It starts with the upstream area with rainfall as input. From that point the model follows the river water via a river, estuary and ends with an ecological model in the estuary which is connected to the sea. The input and output variables in the coupled models therefore mainly consist of river discharges. Other variables are rainfall and evaporation in the river catchment, the salinity in the estuary and some ecological variables (see Table 1).

The final step in the methodology is the connection of the neural networks. The trained neural networks are placed in a
Table 1. Model input and output for neural network.

\begin{tabular}{lllll}
\hline No. & Model & Input & Output & Time step \\
\hline 1 & RR model & $\begin{array}{l}\text { precipitation } \\
\text { and evaporation }\end{array}$ & run-off & 1 day \\
2 & River flow & $\begin{array}{l}\text { run-off } \\
\text { river discharge } \\
\text { and sea level }\end{array}$ & $\begin{array}{l}\text { river discharge } \\
\text { salinity }\end{array}$ & $30 \mathrm{~min}$ \\
3 & Estuary & Secchi-depth & $30 \mathrm{~min}$ \\
\hline
\end{tabular}

cascading manner such that the outputs are connected properly to the inputs. This results in four models that are connected by neural networks. In the coupled scenario, the connections are established without the original software applications.

The four different models have been integrated into one software model of only one application. This results in a model coupling of the four models with a standard technique. This gives the possibility to compare results from the neural network with traditional coupling.

\subsection{Input data and simulations}

The calculation consists of three steps. The first step starts with the coupled system formed by the four models. In cascading order these are the rainfall-runoff model (HBV), the 

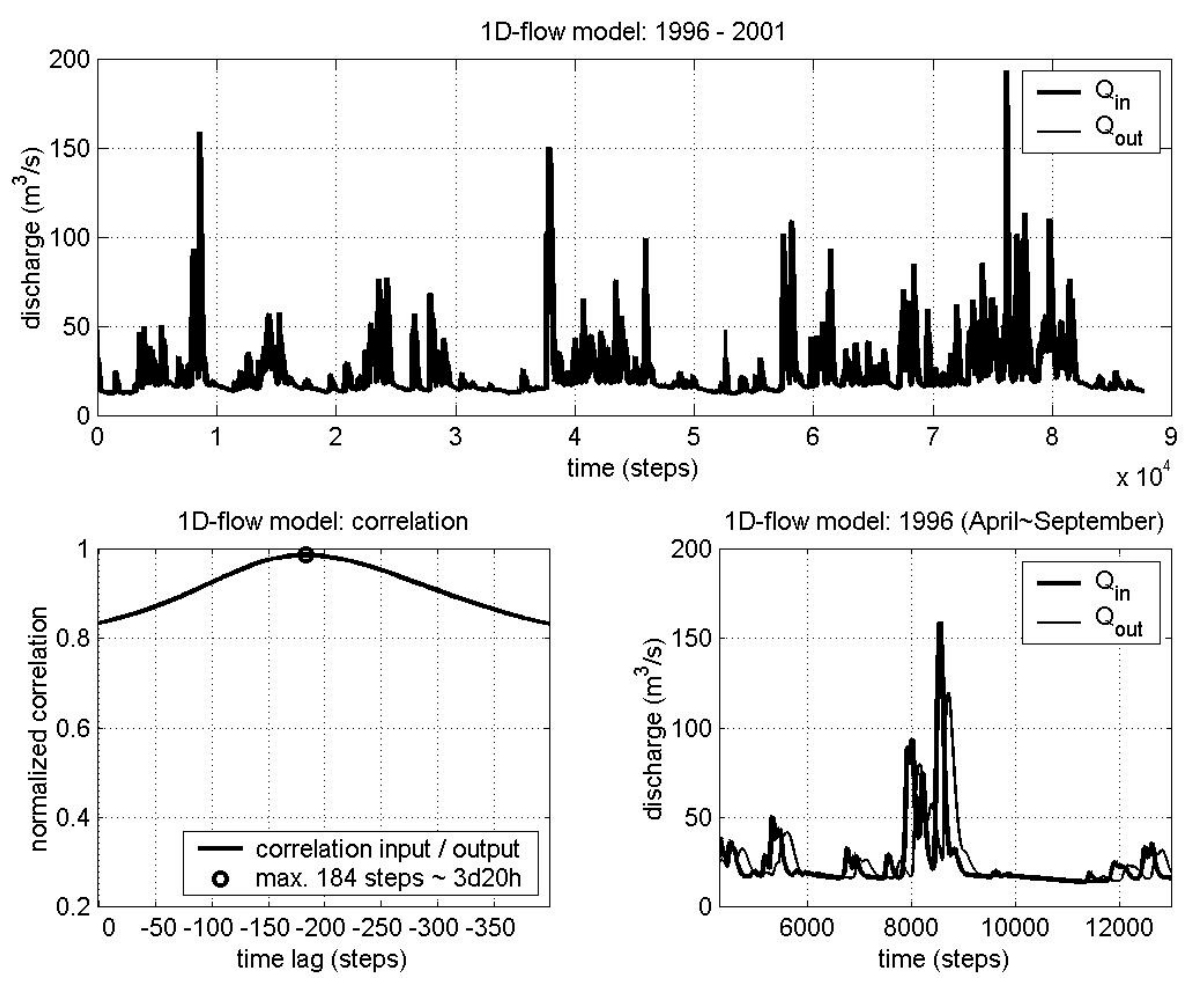

Fig. 2. 1-D-River model, input discharge and neural network prediction (upper), correlation input/output discharge (lower left), input discharge and NN prediction - detail (lower right).

river model, the estuary model and an ecological model with Secchi-depth. Input to this rainfall-runoff model (RR-model) is five years of daily rainfall and evaporation data (see Fig. 1). The downstream boundary condition for the estuary is the sea level with standard tidal movements. The rainfall and evaporation consists of observed values. All other data are calculated by the integrated system. From these data it is possible to construct corresponding input and output data sets for submodels, for example the upstream and downstream discharge values for the river model.

The second step consists of dividing the input and output data for each sub-model into a training set that consists of the first three years and of a test set that consists of the last two years of the data. With a training and test set it is possible to train four neural networks: one for each sub model. With the test set it is possible the express the accuracy of individual neural networks.

In the third step the four models are coupled by connecting the cascading neural network. The results after each cascade can be compared to the results of the integrated system. This makes it possible to follow the progressing error after each model in the cascading coupling.

\subsection{Connection points}

The four models are connected at points that correspond with physically suitable or representative points (see Table 1). The first connection is between the rainfall-runoff model and the river model. This model calculates daily discharges from rainfall and evaporation inputs. The results are connected to the river model at an upstream discharge point. The time step is days.

The river model is a hydraulic model with a time step of half an hour. The discharge from the rainfall-runoff model is the upstream input. The river model ends where the estuary begins. The river discharge downstream of this river is the output of the model. The river is a one-dimensional flow model.

The estuary is an area that starts at the river boundary, with the sea as the downstream boundary. The sea level has a daily pattern. Because it is connected and part of the river system it is also subject to a calculation output step of half an hour. The upstream boundary is the same point as the downstream discharge point of the river. For the estuary a salt intrusion model has been build. The salinity varies with space and with time. For output a point has been selected where both the sea (tidal variation) and the river (seasonal discharge variations) clearly influenced the salinity. The output of the model consist of salinity values at every half an hour.

The ecological model is connected to the salinity values at the same, fixed point along the estuary. Input to this ecological model is the quotient of river water and marine water as simulated by the salt intrusion model. The river water is 
assumed to be muddy and the sea water to be relatively clear. This quotient is input to the Secchi-depth model. The Secchidepth is taken as output and has the same time interval.

\section{Model description}

\subsection{ANNs}

The basic elements of ANNs are neurons that are connected by transfer functions in layers and a network. In mathematical terms a neuron $k$ can be described by writing the following pair of equations (Haykin, 1999):

$u_{k}=\sum_{j=1}^{m} w_{k j} x_{j}$

$y_{k}=\varphi\left(u_{k}+b_{k}\right)$

where $x_{1}, x_{2}, \ldots, x_{m}$ are the input signals, $w_{k 1}, w_{k 2}, \ldots, w_{k m}$ are the synaptic weights of neuron $k ; u_{k}$ is the linear combiner output due to the input signals; $b_{k}$ is the bias and phi (.) is the activation function; and $y_{k}$ is the output signal of the neuron. The sigmoid transfer function is the most common form of activation used. In this function $a$ is the slope parameter and $v$ the local field.

$\varphi(v)=\frac{1}{1+\exp (-a v)}$

A few rules of thumb are available to design an ANN for hydrological modeling (Zijderveld, 2003; Hagan et al., 1996). The ANNs have to be trained to calculate the values of the synaptic weights. A measured or observed data set is necessary with known input and corresponding output values.

\subsection{Rainfall-runoff model}

For the rainfall-runoff model a lumped model of the Alzette Basin, Luxembourg is used (Fenicia et al., 2006). One of the selection criteria is that the size of this basin is sufficient for rainfall-runoff modeling. Large and complex models are not necessary to test model coupling. Many researchers have shown it is possible to simulate a rainfall-runoff model with an ANN (Vos and Rientjes, 2005; Minns and Hall, 1996). Input is rainfall $(P)$ and the potential evaporation $\left(E_{p}\right)$. The output is the downstream discharge $(Q)$. The size of the catchment area is $31 \mathrm{~km}^{2}$. Other model parameters are rainfall correction factor (interception), maximum soil moisture content, limit for potential evapotranspiration, maximum value of capillary flow, recession coefficient, percolation from upper to lower response box and recession coefficient. For a description of the rainfall-runoff model reference is made to Lindström et al. (1997).

\subsection{River model in 1-D-channel flow}

For simulating river flow, the Duflow modeling software is used. There are several publications on simulating hydraulic flow, e.g. Bobovic and Abbott (1997); Dibike (2002); Price et al. (1998); Campolo et al. (1999); Shrestha et al. (2005). Duflow is based on the one-dimensional partial differential equations that describes non-stationary flow in open channels. These equations are the mathematical translation of the laws of conservation of mass and momentum. The equation of conservation of mass reads:

$\frac{\partial B}{\partial t}+\frac{\partial Q}{\partial x}=0$

In which:

$B$ cross-sectional storage area, $Q$ the discharge. The equations are discretized in space and time using the four-point implicit Preissmann scheme. The space between calculation points $\Delta x$ is $3000 \mathrm{~m}$, the calculation time step $\Delta t$ is $30 \mathrm{~min}$.

The equation of conservation of momentum read:

$\frac{\partial Q}{\partial t}+g A \frac{\partial H}{\partial x}+\frac{\partial(\alpha Q v)}{\partial x}+\frac{g|Q| Q}{C^{2} A R}=0$

In wich:

$g$ acceleration due to gravity, $A$ cross-sectional flow area, $H$ water level, $\alpha$ correction factor for non-uniformity of velocity due to advection, $v$ mean velocity (averaged over crosssectinal area), $C$ coefficient of Chézy and $R$ hydraulic radius of the cross-section.

\subsection{Salt intrusion in alluvial estuary}

An estuary is the transition zone between the river and the sea. Alluvial estuaries have movable beds consisting of sediments of riverine and marine origin. The water moving in the estuary can either erode the estuary bed or it can deposit sediments. This results in a dynamic equilibrium situation (Hunkins, 1981; Uncles et al., 1983). In this paper we chose the derivation of the steady state intrusion for the tidal average (TA) model. In the one-dimensional flow model, the dispersion at high water slack $\left(D^{\mathrm{HWS}}\right)$, varies with the tide and river flow (see Fig. 4). The figure shows the variations with tide and flow and the calibration results for low and high river flows. For neural networks it is difficult to combine fast, tidal variations with relatively slow seasonal river discharges. For this reason both a normal estuary model and a "Maximum Salinity" model has been build. The latest only simulates the maximum salinity during a tidal period: simulation of detailed, tidal motions by the neural network is not necessary. Figure 8 shows the results when the models are coupled to represent the maximum daily salinity values. $D_{0}^{H W S}$ is the high water slack dispersion at the downstream boundary. The salt intrusion model was developed by Savenije (1989, 1993b, 2005):

$\frac{S-S_{f}}{S_{0}-S_{f}}=\left(\frac{D}{D_{0}}\right)^{\frac{1}{K}}$ 

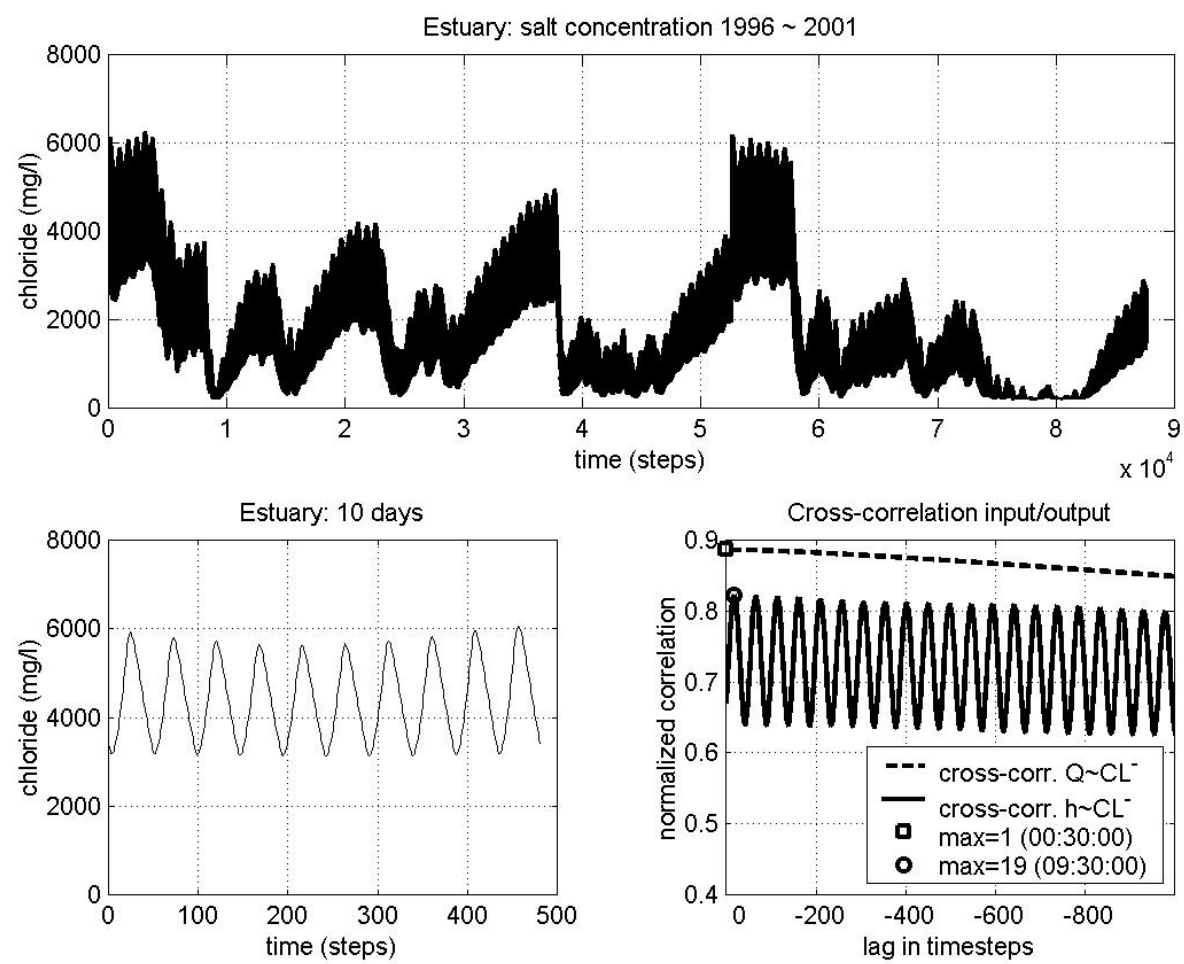

Fig. 3. Salinity in estuary model, neural network salinity prediction (upper), detail with daily tidal influence (lower left), correlation input discharge and input water level with salinity output (lower right).
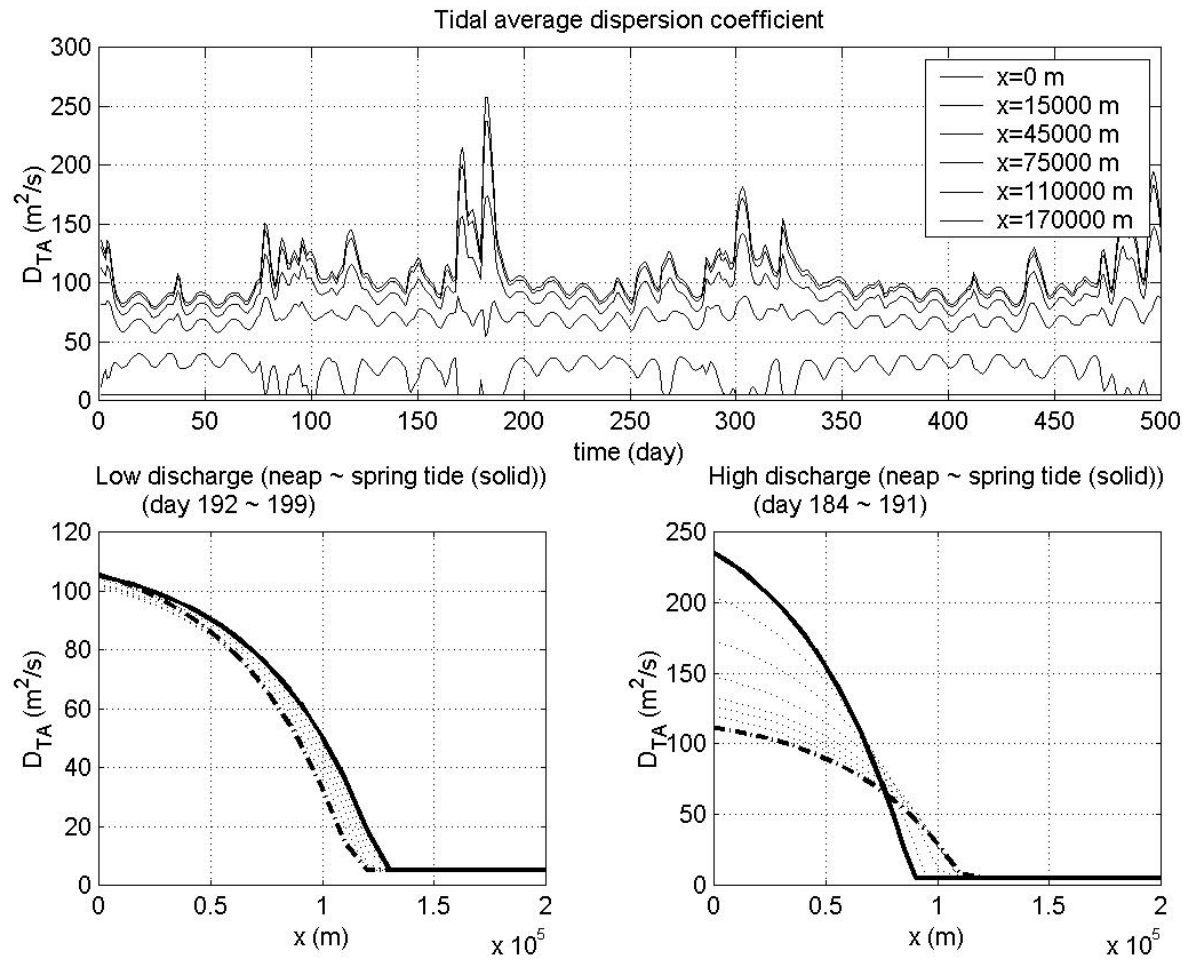

Fig. 4. Dispersion coefficient in estuary, tidal average dispersion for validation (upper), tidal average dispersion along estuary $\mathrm{x}$-axis for calibration low river flows (lower left), tidal average dispersion along estuary x-axis for calibration of high river flows (lower right). Spring tide is the bold, solid line and neap tide the bold, dashed line. 


$$
\begin{aligned}
& \frac{D^{\mathrm{HWS}}}{D_{0}^{\mathrm{HWS}}}=1+\frac{K a Q_{f}}{D_{0}^{\mathrm{HWS}} A_{0}}\left(\exp \left(\frac{x}{a}\right)-1\right) \\
& D_{0}^{T A}=D^{\mathrm{HWS}}(E / 2) \cdot \exp \left(-\frac{E}{2 a}\right)
\end{aligned}
$$

where $K$ is the Van der Burgh's coefficient, $S, S_{0}$ and $S_{f}$ the salinity, salinity at the estuary mouth and fresh water salinity respectively. $Q_{f}$ is the fresh water discharge which in $x$ are negative since they are points upstream, $A_{0}$ is the tidal average cross-sectional area at the estuary mouth and $a$ is the cross-sectional area convergence length. Furthermore the predictive equation for the downstream boundary condition and the shape function apply:

$$
\begin{aligned}
& \frac{D_{0}^{H W S}}{v_{0} h_{0}}=1440 \frac{E}{a} \sqrt{N_{R}} \\
& E=H \frac{a}{h_{0}} \cos (\epsilon) \\
& N r=\frac{\Delta \rho}{\rho} \frac{g h}{A_{0}} \frac{Q_{f} T}{E_{0} v_{0}^{2}}
\end{aligned}
$$

With $E$ the tidal excursion, $\epsilon$ the phase difference between high water (HW) and high water slack (HWS), $T$ tidal period and $v_{0}$ tidal velocity amplitude at the estuary mouth.

\subsection{Secchi-depth}

The under water light climate is an important factor for the development of the aquatic eco-system. Growth of algae and water plants is strongly dependent on the availability of light under water. The contribution of optical active components to adsorption and diffusion of light is linear related to concentration of components. Total extinction of light for plants and algae in the most important wave length (400-700 nm) is described by the extinction coefficient $K_{d}$. The visibility is expressed and measured as the Secci-depth $\left(d_{S}\right)$ Blom (1992):

$d_{S}^{-1}=d_{S 0}^{-1}+\beta_{h} \cdot E_{\mathrm{abs}(380)}+\beta_{a} \cdot C_{\mathrm{chla}}+\beta_{d} \cdot C_{\mathrm{det}}+\beta_{m} \cdot C_{\min }($

in which:

$d_{S 0}$ background Secchi-depth, $E_{\text {abs(380) }}$ absorption of light dissolved material at $380 \mathrm{~nm}, C_{\text {chla }}$ concentration of cholorofyl-a, $C_{\text {det }}$ concentration of suspensive organic matter, $C_{\min }$ concentration of suspensive mineral matter. And, $\beta_{h}$ contribution of humus acids to inverse Secchi-depth, $\beta_{a}$ contribution of chlorofyl-a to inverse Secchi-depth, $\beta_{d}$ contribution of detritus to inverse Secchi-depth, $\beta_{m}$ contribution of floating matter to inverse Secchi-depth.

We assumed the concentration of suspensive organic matter $C_{\mathrm{det}}$ is linear related to the quotient of muddy river water and the saline sea water $\left(\frac{S_{f}}{S}\right)$. This Secchi-depth model is implemented as a water quality model in the 1-D-flow model.

\subsection{Training}

The most important step is to train the ANNs. The data set should contain enough physical events such as high and low flows. If this is not the case, there is the possibility to create artificial training data in a systematical way based on physical features such as mean sea level, maximum flow, amplitude at estuary mouth, typical time variations etc. Many of these parameters can be subtracted from the conceptual model. Basic statistical parameters of a data source also give shape to the input space. Selecting correct data sets is important (Doan et al., 2005). In Kamp and Savenije (2006) the authors showed additional optimisation of the original artificial data is possible in combination of a Genetic Algortihms (GA). The GA constructs a new training set by selecting different subsets from the original training set resulting in better performance of the ANN. In this paper this methodology was not applied because a daily dataset of five years was available.

ANNs consist of an input layer, one or several hidden layers and an output layer. Each layer consists of one or more neurons and all neurons of two successive layers are connected. Every connection gives a signal to the next layer multiplied by a factor. The neurons transfer this signal with a transfer function. ANNs are described in detail by Haykin (1999).

\section{Simulations}

\subsection{Case study}

In the case study the simulation results of four separate hydrological models will be mimicked by neural networks. The hydrological models were calibrated with the rainfall data from the Alzette basin with five years of rainfall and evaporation events. The case study is the simulation of four coupled models that represents parts of a hydrological cycle. The resemblance between the model and reality is investigated during validation. To train the neural networks it was necessary to split the available data into training data (the first three years) and testing data (last two years). From many runs with neural networks it showed that the use of crossvalidation did not improve the calculation and was not used in the final simulation.

\subsection{Design and training}

For every neural network we used an input layer and two hidden layers. All layers are feedforward layers. The model input is presented to the input layer of the neural network by a time stepped delay line in which each input is duplicated and delayed several times. The first hidden layer consists of 7 or 3 neurons depending on training results. The second hidden layer consists of three hidden layers. There are not many design rules which leaves large freedom for the designer. For 
each neural network several configurations of neurons and layers have been tested by trial-and-error and using experience. All transfer functions are sigmoid functions (Eq. 3) except for the output layer which has a linear transfer function. The trainings function is Levenberg-Marquardt back propagation. A stepped delay line is used to simulate flow dynamics. In a stepped delay line the input at time $t$ until $n$ steps in history $Q_{t-n}$ form the ANN's input:

$\mathbf{Q}=\left(\begin{array}{c}Q_{t-1} \\ Q_{t-2} \\ \vdots \\ Q_{t-n}\end{array}\right)$

To assess the length of the delay line, a graph of the crosscorrelation between input and output signals can be made. This graph provides the correlation of a delayed input vector and the (target) output signal. Cross validation for early stopping is not used. The average number of epochs or calculation runs for the training phase is 50. All design and train parameters are optimised and based on the authors expert knowledge. For testing the root mean squared error $\left(E_{\mathrm{RMSE}}\right)$ is used. Also the Nash-Sutcliffe efficiency index $\left(I_{N S}\right)$ is used to assess the predictive power of hydrological models, and the Pearson's r-squared statistics $\left(R^{2}\right)$ for measurement of high flows are used:

$$
\begin{aligned}
& E_{\mathrm{RMSE}}=\sqrt{\frac{\sum_{i=1}^{n}\left(Q_{i}-\widehat{Q}_{i}\right)^{2}}{n}} \\
& I_{N S}=1-\frac{\sum_{i=1}^{n}\left(Q_{i}-\widehat{Q}_{i}\right)^{2}}{\sum_{i=1}^{n}\left(Q_{i}-\bar{Q}_{i}\right)^{2}} \\
& R^{2}=\left[\frac{\sum_{i=1}^{n}\left(Q_{i}-\bar{Q}_{i}\right) \cdot\left(\widehat{Q}_{i}-\widetilde{Q}_{i}\right)}{\sqrt{\sum_{i=1}^{n}\left(Q_{i}-\bar{Q}_{i}\right)^{2} \cdot \sum_{i=1}^{n}\left(\widehat{Q}_{i}-\widetilde{Q}_{i}\right)^{2}}}\right]^{2}
\end{aligned}
$$

where $Q_{i}$ is the observed value, $\widehat{Q}_{i}$ is the modelled value and $\bar{Q}_{i}$ is the mean of the observed data and $\widetilde{Q}_{i}$ is the mean of the modelled data. In the conjunction of the neural network models the observed values are the values obtained from other models.

\subsection{Rainfall-runoff model}

For the Alzette basin (Pfister et al., 2005) daily time series are available for five years (1996-2001) for precipitation and potential evaporation (see Fig. 1). A conceptual rainfallrunoff model was available with calibrated parameters. From the cross-correlation graph of precipitation and the potential evaporation, it appears that a history of six delayed time steps is sufficient.

The feedforward neural network consisted of one input layer and two hidden layers with both three neurons having both sigmoid transfer functions. The precipitation and the potential evaporation were used as input for the neural network. Both input parameters were delayed by a time delay line of six days. Target values are the downstream river discharges. However, training and testing the ANN showed a more basic problem. The training set should contain enough high flows. In the training set only a few high floods occurred. No extreme high flood occurred in the training and test set. This resulted in a poor prediction of only 0.66 (see Fig. 6). This figure shows all model results as predicted by the neural networks in uncoupled conditions. Another difficulty for neural networks is the fact that a rainfall-runoff model has different model states. The response in wet situations is much quicker than in dry periods, which are difficult training conditions. In Campolo et al. (1999) the past flow values were added in input for distinguishing between wet and dry conditions. In additional experiments the rainfallrunoff model past flow values were added resulting in better predictions, e.g. RMSE of 3.4 instead of $11.5 \mathrm{~m}^{3} / \mathrm{s}$, see Table 3 column (RMSE $Q_{t-1}$ ). The $R^{2}$ improved from 0.51 to 0.97 . This approach should however be exercised with care. The autoregressive model component can become too dominant, resulting in lagged model forecasts (Vos and Rientjes, 2005).

Additional attention has to be payed to different time scales between the models. The rainfall-runoff model for example simulates daily discharge values, while the flow model has a time step of $30 \mathrm{~min}$. Therefore the standard discharge points in Duflow were used to simulate the HBV model results as flow boundaries.

\subsection{River model in 1-D-channel flow}

The largest river section connects the inflow from the rainfall-runoff model to the inflow of the estuary and has an average slope $(I)$ of $1.2 \times 10^{-4} \mathrm{~m}^{-1}$. The distance between the input and output point is $336 \mathrm{~km}$. The cross sectional profile is $20 \mathrm{~m}$ wide $(B)$, rectangular and uniform with no flooding area's for water storage $\left(B_{S}\right)$. The discharge $(Q)$ is $13 \mathrm{~m}^{3} / \mathrm{s}$ at low flow, $50 \mathrm{~m}^{3} / \mathrm{s}$ at high flow and $100-150 \mathrm{~m}^{3} / \mathrm{s}$ in extreme situations. The water depth $(h)$ is $1.6 \mathrm{~m}$ and the (steady state) water velocity $(\bar{v})$ at the top of the high water wave $(\partial h / \partial t=0)$ can be described as a steady state flow according to Manning-equation:

$\bar{v}=\frac{1}{n} h^{2 / 3} \sqrt{I}$

If we assume $Q=\bar{v} B_{s} h$ and substitute it in the law of conservation (Eq. 4), the high water wave velocity is (Savenije, 2001):

$c=\frac{5}{3} \frac{B_{s}}{B} \frac{1}{n} h^{2 / 3} \sqrt{I}=\frac{5}{3} \frac{B_{s}}{B} \bar{v}$

With $n=0.025$ (Manning) for clean, straight and uniform river bed, we find a theoretical $c=1.03 \mathrm{~m} / \mathrm{s}$, and from the 1-D-flow simulation model we find a wave celerity of 

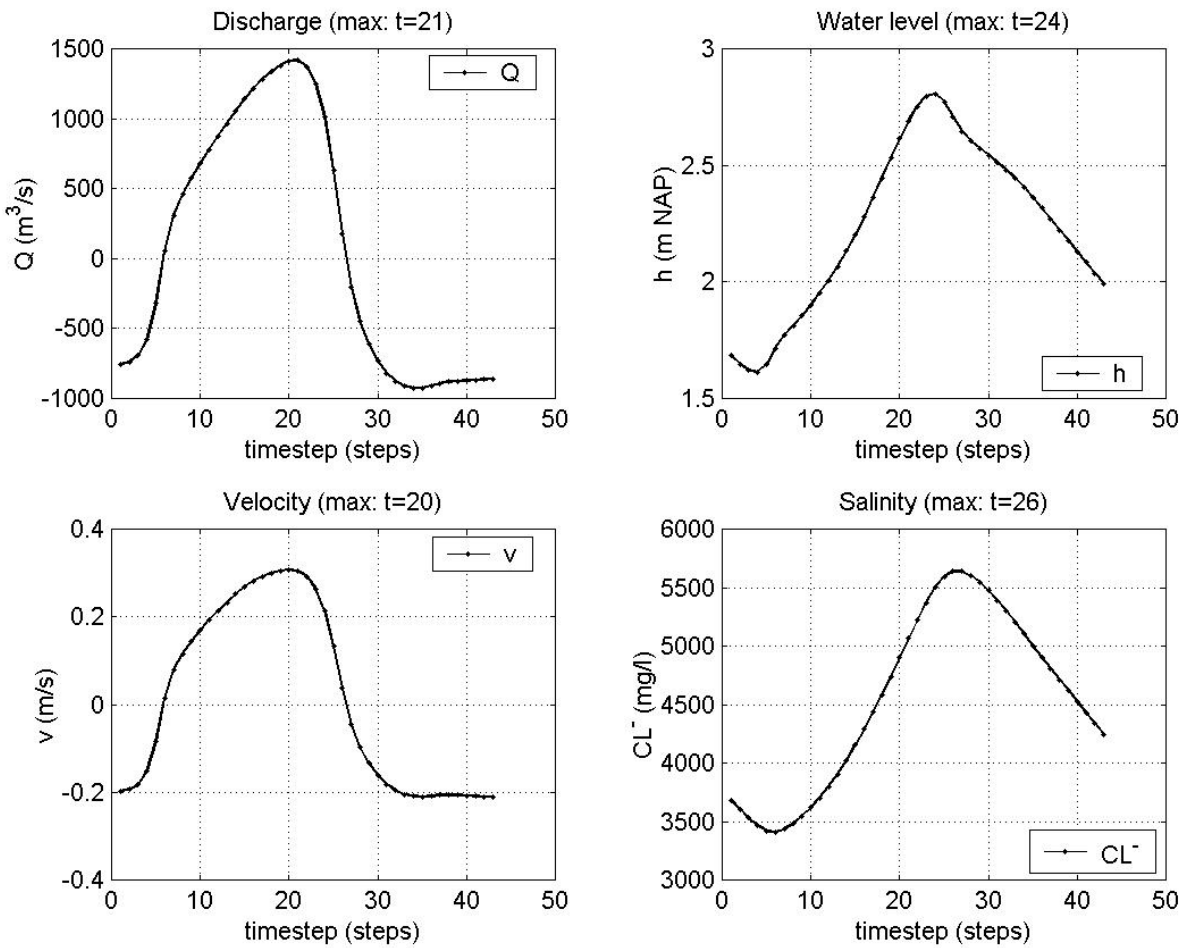

Fig. 5. Tidal movement in point (SCH00004) for calibration of discharge (upper left), water level (upper right), velocity (lower left) and salinity (lower right).
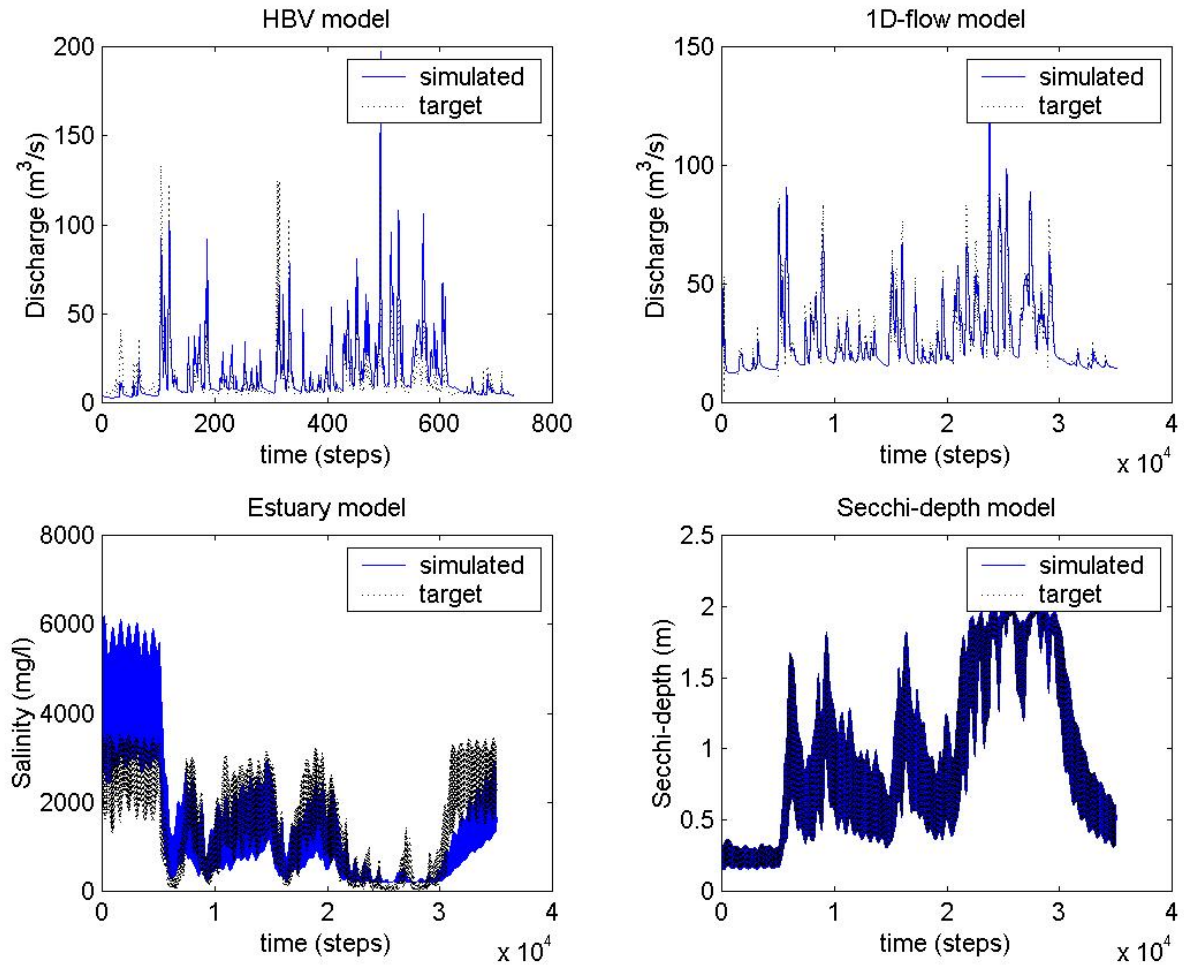

Fig. 6. Neural network predictions of four separated models. Validation results from RR-model with river discharge (upper left), river discharge from river model (upper right), salinity from estuary model (lower left) and Secchi-dept from ecological model (lower right). 

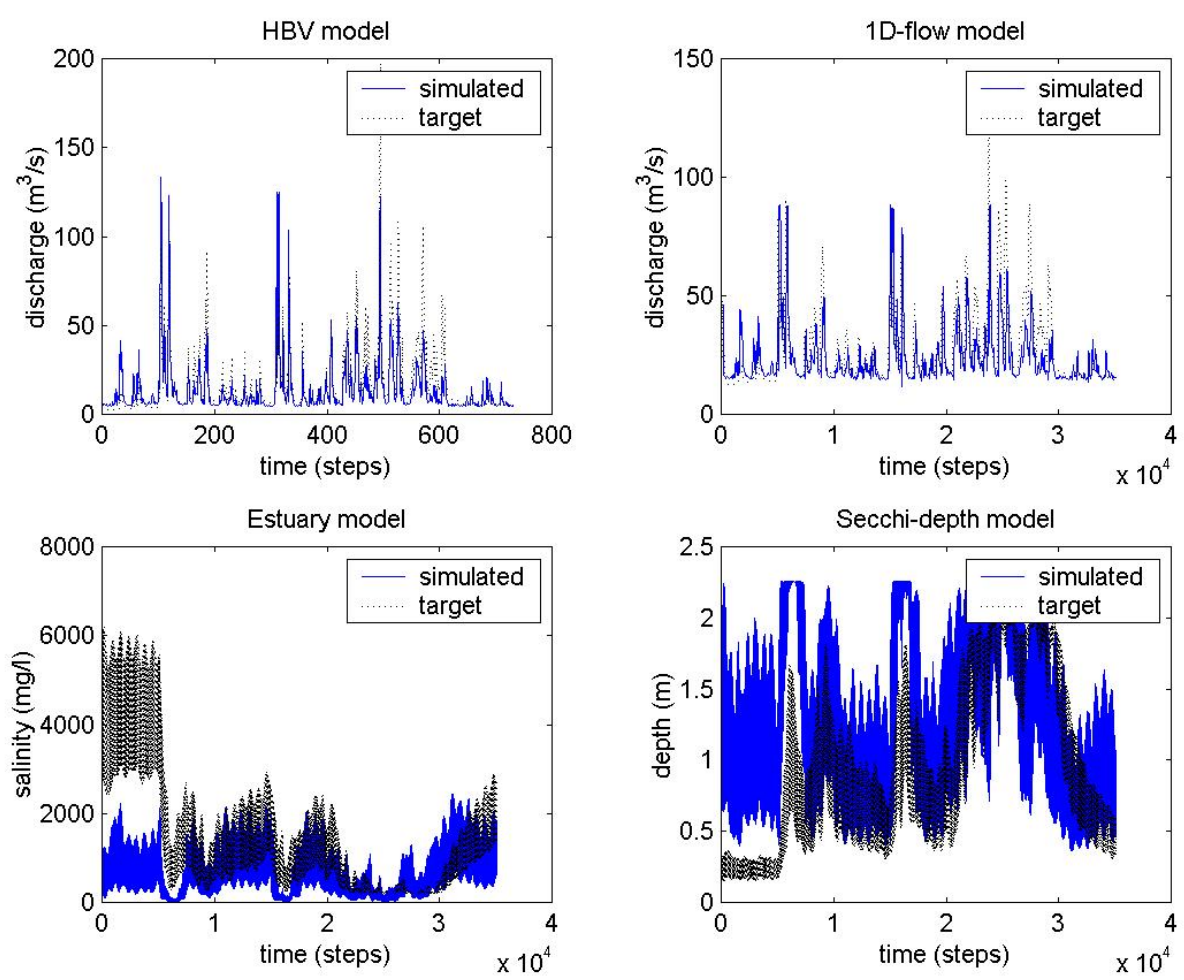

Fig. 7. Neural network predictions of four models in cascading coupled simulation. Results from RR-model with river discharge (upper left), river discharge from river model (upper right), salinity from estuary model (lower left) and Secchi-dept from ecological model (lower right).

$c=\frac{\Delta x}{\Delta t}=1.00 \mathrm{~m} / \mathrm{s}$. A river flood upstream arrives 3 days and $20 \mathrm{~h}$ later in the downstream area (see Fig. 2). This is important for the stepped delay line used for training (Eq. 13).

The feedforward neural network consisted of one input layer and two hidden layers with five and three neurons having both sigmoid transfer functions. The input for the neural network in the connected model is the upstream river discharge which was simulated with a delay line of two time steps delayed by 3 days and $20 \mathrm{~h}$. The output of this neural network model is the downstream river discharge. The output will be connected to the salt intrusion model.

The results fitted quite well and resulted in RMSE $=4.4 \mathrm{~m}^{3} / \mathrm{s}$ and an efficiency of $R^{2}=0.92$ (see Fig. 6). The results are good because the hydro-graph was symmetric and showed little deformation. In situations of large water storage and non-uniform cross-sectional profile this is not the case.

The time step of the rainfall-runoff model is days, while the time step of the dynamic flow model is half an hour. This means that 48 steps of the flow model corresponds to one time step of the rainfall-runoff model. Without interpolation the signal from the rainfall-runoff model will have sudden changes after a period of one day. It is better for a dynamic, hydraulic system to prevent sudden steps and numerical shock waves. Hence, we used interpolated values on the intermediate time steps.

\subsection{Salt intrusion in alluvial estuary}

The salt intrusion is modeled in an estuary that connects the 1-D river flow with the sea. The upstream boundary of the alluvial estuary is the fresh river inflow. Figure 5 shows the calibration results for the tidal characteristics in the estuary. Downstream the MSL is $2.0 \mathrm{~m}$ with an average amplitude of $1.25 \mathrm{~m}$. The geometric profile is wide at the estuary mouth and small at the river mouth. The width varies as an exponential function with distance. The bottom level is constant $(5.0 \mathrm{~m})$. These are conditions for alluvial estuaries that fit the model as described by Savenije (Sect. 3.4).

The feedforward neural network consisted of one input layer and two hidden layers with five and three neurons having both sigmoid transfer functions. Input is the fresh river from the river. The second input is the sea level at the downstream area where the river disperses into the sea. The river discharge is delayed by 12 calculation steps corresponding with $6 \mathrm{~h}$. The output of the salt intrusion model is the salinity at a point $120 \mathrm{~km}$ upstream from the estuary mouth. The simulation results are shown in Fig. 4. In this point the salinity is influenced by both the fresh river discharge and the tidal movement at the estuary mouth (see Fig. 3). In the upper plot of this figure the performance corresponds with river flow variations. The tidal influence (lower left) effects the short term. This is also shown in the correlation graphs (lower right). 

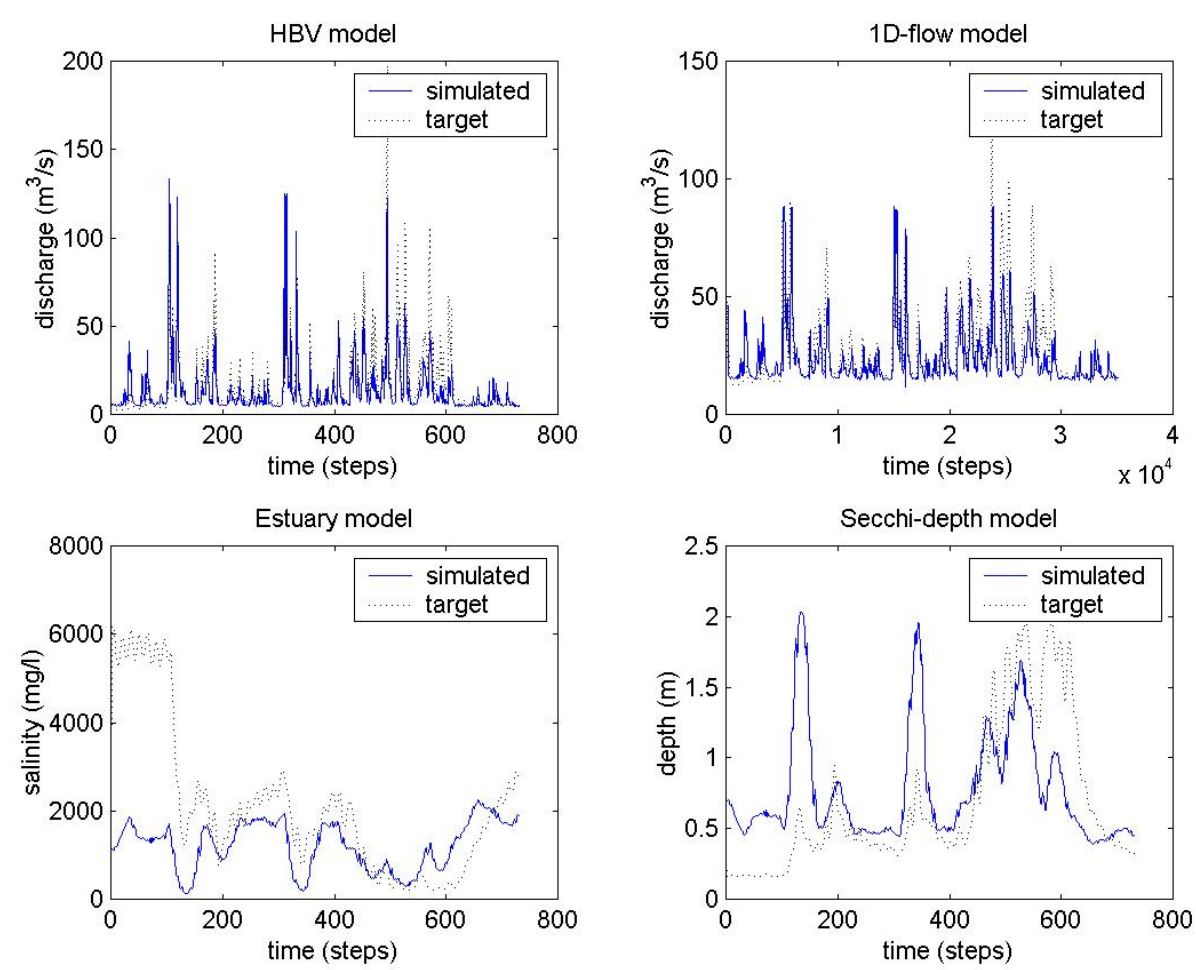

Fig. 8. Neural network predictions of four models in cascading coupled simulation with improved estuary model. Results from RR-model with river discharge (upper left), river discharge from river model (upper right), salinity from estuary model trained on maximum values excluding daily, tidal variations (lower left) and Secchi-dept from ecological model (lower right).

Table 2. Simulation results of single models.

\begin{tabular}{llll}
\hline Model & RMSE & $R^{2}$ & RSQR \\
\hline RR-model & $11.4 \mathrm{~m}^{3} / \mathrm{s}$ & 0.51 & 0.66 \\
RR-model $\left(Q_{t_{0,-1}}\right)$ & $3.4 \mathrm{~m}^{3} / s$ & 0.97 & 0.97 \\
River flow & $4.4 \mathrm{~m}^{3} / \mathrm{s}$ & 0.92 & 0.93 \\
Estuary & $837 \mathrm{mg} / 1$ & 0.38 & 0.62 \\
Secchi-depth & $0.004 \mathrm{~m}$ & 0.99 & 0.99 \\
\hline
\end{tabular}

The first process, the tidal movement, has a time period of one day. The variations of the discharge includes several days and is a much slower process. It is important to understand that it is difficult for one ANN to simulate both time scales in one training. To improve the performance the moving average value of discharge is used for input. This gives better results but introduces a larger error in the starting period when the model has to build a "history".

Training the ANN was difficult and gave poor results. Introducing a moving history was necessary but did not give satisfying predictions. Although both the sea level and river discharge have an effect on salinity, it is difficult to separate these two processes.

\subsection{Secchi-depth}

The Secchi-depth is an indication for the light penetration under water. This value is in our model directly derived from the quotient of river and sea water. We assumed this is an indication of dissolved matter in the water column. Except for salinity, all other parameters are assumed constant and there are no external variables distinguished in this model. If the salinity is high, the assumption is that there is relatively much sea water hence less muddy river water. In that situation the concentration of dissolved material is low and the visibility is high. This results in a Secchi-depth which is proportional with salinity.

The feedforward neural network consisted of one input layer and two hidden layers with seven and three neurons having both sigmoid transfer functions. The salinity in the estuary at the fixed point $120 \mathrm{~km}$ upstream from the estuary mouth is input data for the neural network. This point is connected to the output of the salt-intrusion model. Output of the model is the Secchi-depth value. This is calculated at the same fixed point in the model. In this process there is no time lag and the time delay line is not used. Training a neural network with no time lags can result in a high accuracy. It can be compared to universal function approximation. In this specific model the neural network also showed accurate results with $R^{2}=0.99$ (see Fig. 6). 
Table 3. Simulation results coupled models.

\begin{tabular}{llll}
\hline Model & $\begin{array}{l}\text { Coupled models } \\
\text { (RMSE) }\end{array}$ & $\begin{array}{l}\text { Coupled models with } \\
\text { RR-model } Q_{t_{0,-}} \text { (RMSE) }\end{array}$ & $\begin{array}{l}\text { Progressive error. Single ANNs with } \\
\text { input: } Q_{t_{0,-1}}, P, E_{p}, h_{\text {sea }} \text { (RMSE) }\end{array}$ \\
\hline RR-model & $11.4 \mathrm{~m}^{3} / \mathrm{s}$ & $3.4 \mathrm{~m}^{3} / \mathrm{s}$ & $3.4 \mathrm{~m}^{3} / \mathrm{s}$ \\
River flow & $13.7 \mathrm{~m}^{3} / \mathrm{s}$ & $11.3 \mathrm{~m}^{3} / \mathrm{s}$ & $12.8 \mathrm{~m}^{3} / \mathrm{s}$ \\
Estuary & $1424 \mathrm{mg} / \mathrm{l}$ & $1148 \mathrm{mg} / 1$ & $1695 \mathrm{mg} / 1$ \\
Secchi-depth & $0.636 \mathrm{~m}$ & $0.26 \mathrm{~m}$ & $0.59 \mathrm{~m}$ \\
\hline
\end{tabular}

\subsection{Model coupling and results}

Models sharing the same physical variables, for example water levels or discharges, can be connected if these variables are available for both input and output. In this paper we used a cascading model coupling with a line-up of four hydrological sub-systems. The line-up of models is (1) the rainfallrunoff model producing discharge, (2) the 1-D-flow model for the river, (3) the salt intrusion model and (4) the Secchidepth model. The rainfall-runoff model produces discharge as input to the river model; the river model generates discharge to the estuary; and the estuary model generates salinity for the Secchi-depth model. The latter is a water quality model for light penetration in ecological processes.

For each model an ANN has been designed and trained. Each single model has been tested before use in the cascading simulation. The results of the individual ANNs are presented in Table 2. In general the ANNs are able to simulate the model results. For the rainfall-runoff simulation an improved model has been developed which also uses previous flow values as input. These two rainfall-runoff models correspond with the first two columns of Table 3. This table and Fig. 7 present the results of the coupled models in cascade. Not all predictions are accurate, due to several problems. In the river model, the flow can normally be well simulated if it has a uniform cross-section and no flooding area's. In this model however it was difficult to obtain accurate results because of the lower accuracy of the output of the rainfall-runoff model. In addition, the ANN of the estuary could hardly distinguish the two processes with different time scales. The Secchidepth, finally, is proportional to the salinity without any time delay and gave perfect results. The final, coupled model performs not very good, because errors are accumulated in the cascading modeling scenario. These errors mainly stem from the rainfall-runoff and salinity models. One way to test if the accumulation of errors is a dominant factor, is to design single ANNs covering two or more sub-systems at once. It is for example interesting to see if a single neural network can predict the outcome of the river model, if it only uses rainfall and evaporation data as input. The results of three additional neural networks are presented in the third column of Table 3. The results are comparable for the river model, however they get worse if the estuary model is implemented. This analysis shows that the accumulation of errors are not caused by ANN design and training errors, but by the ANNs having difficulty to correctly represent the physical processes.

\section{Conclusions}

In this research ANNs represent four hydrological models. The ANNs were trained with a data set of three years and they were tested with a data set of two years. The ANNs have been coupled in a cascading set-up and compared to an integrated hydrological model. We found that it is possible to use ANNs for model coupling. The ANNs were capable to simulate the output of the different model components. The individual ANNs were tested and three of the four resulted in good results. However, the final model results are as accurate as the weakest link in the model chain. In this model simulation the salt intrusion model was not accurate enough. The ANNs could simulate the tidal movement (short term) but simulated the salt-intrusion (long term) inaccurately. Additional research has to be done on a method that can separate the short and long term processes for the salt-intrusion in an estuary.

We can conclude that model coupling as such has proved to be feasible and efficient, however the overall accuracy of four coupled models was not sufficient due to the poor performance of the ANN in mimicking the salt-intrusion model.

Edited by: D. Solomatine

\section{References}

Blom, G.: Licht onder water, Het belang van extinctiemetingen, LU Wageningen, Vakgroep Waterkwaliteitsbeheer en Aquatische Oecologie, Wageningen, 1992.

Babovic, V. and Abbott, M. B.: The evolution of equations from hydraulic data: Part I -Theory, J. Hydraul. Res., 35(3), 1-14, 1997.

Campolo, M., Andreussi, P., and Soldati, A.: River flood forecasting with a neural network model, Water Resources Research, 35(4), 1191-1197, 1999. 
Dibike, Y. B.: Model Induction from Data: Towards the next generation of computational engines in hydraulics and hydrology, IHE Delft, Delft, 2002.

Doan, C. D., Liong, S. Y., and Karunasinghe, D. S. K.: Derivation of effective and efficient data set with subtractive clustering method and genetic algorithm, J. Hydroinform., 7, 219-233, 2005.

Fenicia, F., Savenije, H. H. G., Matgen, P., and Pfister, L.: A comparison of alternative multiobjective calibration strategies for hydrological modeling, Water Resour. Res., 43, W03434, doi:10.1029/2006WR005098.

Hagan, T., Demuth, H. B., and Beale, M. H.: Neural Network Design, PWS Pub. Co., Boston, 1996.

Haykin, S.: Neural Networks, a comprehensive foundation, Prentice Hall, New Jersey, 1999.

Hunkins, K.: Salt dispersion in the Hudson Estuary, J. Phys. Oceanogr., 11(5), 729-738, 1981.

Kamp, R. G. and Savenije, H. H. G.: Optimising training data for ANNs with Genetic Algorithms, Hydrol. Earth Syst. Sci., 10, 603-608, 2006, http://www.hydrol-earth-syst-sci.net/10/603/2006/.

Koudstaal, R., Rijsberman, F. R., and Savenije, H. H. G.: Water and Sustainable Development, Natural Resources Forum, 16, 4, 277-290, 1992.

Lindström, G., Johansson, B., Persson, M., Gardelin, M., and Bergström, S.: Development and test of the distributed HBV-96 hydrological model, J. Hydrol., 201, 272-288.

Minns, A. W. and Hall, M. J.: Artificial neural networks as rainfallrunoff models, Hydrolog. Sci. J., 41, 3, 399-417, 1996.

Pfister, L., Wagner, C., Vansuypeene, E., Drogue, G., and Hoffmann, L.: Atlas climatique du Grand-Duche de Luxembourg, Johnen-druck GmbH Co, Bernkastel-Kues, 2005.
Price, R. K., Samedov, J. N., and Solomatine, D. P.: An artificial neural network model of a generalised channel network, Proc. of 3rd Int. conf. on Hydroinformatics, Copenhagen, 1998.

Savenije, H. H. G.: Salt intrusion model for High Water Slack, Low Water Slack and Mean tide, on spreadsheet, J. Hydrol., 85, 87109, 1989.

Savenije, H. H. G.: Hatsi-kD, Voortplanting van hoogwatergolven in rivieren, Stromingen, 7(4), 45-47, 2001.

Savenije, H. H. G.: Predictive model for salt intrusion in estuaries, J. Hydrol., 148, 203-218, 1993b.

Savenije, H. H. G.: Salinity and Tides in Alluvial Estuaries, 208, Elsevier, 2005.

Shrestha, R. G., Theobald, S., and Nestmann, F.: Simulation of flood flow in a river system using artificial neural networks, Hydrol. Earth Syst. Sci., 9, 313-321, 2005, http://www.hydrol-earth-syst-sci.net/9/313/2005/.

Stowa: Duflow Reference Manual, Stowa, version 3.3, 7-10, 2002.

Uncles, R. J., Bale, A. J., Howland, R. J. M., Morris, A. W., and Elliott, R. C. A.: Salinity in surface water in partially-mixed estuary, and its dispersion at low run-off, J. Phys. Oceanogr., 11(5), 729-738, 1981.

Vos, N. J. de and Rientjes, T. H. M.: Constraints of artificial neural networks for rainfall-runoff modeling: trade-offs in hydrological state representation and model evaluation, Hydrol. Earth Syst. Sci., 9, 111-126, 2005, http://www.hydrol-earth-syst-sci.net/9/111/2005/.

Zijderveld, A.: Neural network design strategies and modeling in hydroinformatics, Ph.D. thesis, Delft University of Technology, Delft, 2003. 